Categoria

Trabalho Acadêmico / Artigo Completo

\title{
RELAÇÃO PESO - COMPRIMENTO COM A INFESTAÇÃO DE PEIXES POR NEMATODAS
}

\author{
Marcos Vinicius Nunes ${ }^{1}$
}

Odete Rocha $^{2}$

José Roberto Verani ${ }^{3}$

RESUMO: Os peixes de água doce podem contrair diversos parasitas por meio do consumo de organismos infectados. As larvas de nemátodos de espécies do gênero Contracaecum são frequentemente encontradas em muitas espécies de peixes, dentre os quais o acará, Geophagus brasiliensis. Este serve de hospedeiro intermediário e paratênico. O objetivo do presente trabalho foi determinar o índice de parasitismo pelo nematoda Contracaecum sp em uma população de $G$. brasiliensis, verificando se existe relação entre o grau de infestação e as características do hospedeiro, como o tamanho e peso dos indivíduos. Os resultados mostraram que não existe relação entre a intensidade média de infecção e o comprimento ou peso dos peixes. Também não houve diferenças significativas entre a prevalência de infestação por Contracaecum em G. brasiliensis entre os diferentes períodos climáticos, embora tenha havido uma tendência à maior infestação dos acarás por este parasita em janeiro (período chuvoso).

Palavras-chave: Parasitismo. Peixes. Sazonalidade.

\footnotetext{
${ }_{1}^{1}$ Biólogo, Universidade Federal de São Carlos. vnnybio@hotmail.com.

${ }^{2}$ Bióloga, Universidade Federal de São Carlos. doro@ufscar.br.

${ }^{3}$ Agrônomo, Universidade Federal de São Carlos. verani@ufscar.br.
} 
Volume 8, Número 2, 2012

Bacias Hidrográficas, Planejamento e Gestão dos Recursos Hídricos

\section{INTRODUÇÃO}

As formas adultas das espécies do gênero Contracaecum Railliet \& Henry,1912 são parasitos encontrados no estômago ou intestino delgado, preferencialmente, em aves piscívoras (VICENTE et al., 1985; TORRES et al., 2000, MARTINS et al., 2005), nas formas larvais, são encontrados em muitas espécies de peixes, apresentando baixa especificidade quanto ao hospedeiro intermediário (MORAVEC, 1998, PAVANELLI et al.,2004). Conforme Moravec (1998) as larvas de Contracaecum ficam aderidas ao fígado ou na cavidade abdominal (livre ou encapsulada) dos peixes.

Os peixes contraem o parasita pela predação de outros organismos infectados como copépodos, gastrópodos, celenterados, ctenóforos e peixes menores ou a larva no seu estágio de vida livre, deste modo, a quantidade de parasita por hospedeiro aumenta de acordo com o tamanho e idade do peixe (GUIDELLI et al., 2003).

O Geophagus brasiliensis (Quoy \& Gaimard 1824) (Peciformes, Cichlidae) é uma espécie encontrada desde a bacia Amazônica até a bacia do Plata. O G. brasiliensis faz parte de uma complexa cadeia trófica, sendo presa de outros peixes, aves e mamíferos aquáticos e predadora de diferentes espécies de invertebrados e também serve de hospedeiro intermediário e paratênico de diferentes espécies de helmintos, entre as quais o Contracaecum (MADI, 2005).

O presente estudo teve o objetivo de determinar o índice de parasitismo no $G$. brasiliensis pelo nematoda Contracaecum, e verificar a relação entre o número de parasitas e o tamanho e peso dos indivíduos.

\section{MATERIAL E METODOS}


A coleta foi realizada na represa do Fazzari que é um pequeno reservatório situado na Latitude $21^{\circ} 58^{\prime} S$ e Longitude $47^{\circ} 53^{\prime} \mathrm{O}$, formado pelo represamento do córrego do Fazzari. A represa do Fazzari tem uma área de 1,30 há e profundidade média de 1,5m, suas margens são protegidas por vegetação típica do cerrado (ALBUQUERQUE, 1990).

Foram realizadas 4 campanhas (julho e outubro de 2010 e janeiro e abril de 2011). As coletas foram realizadas com a utilização de diferentes artefatos de pesca: tarrafa, redes de arrasto, redes de espera com diferentes tipos de malha. Os indivíduos coletados foram sedados com óleo de cravo e fixados em formol $10 \%$ ainda no campo, colocados em sacos plásticos contendo as informações sobre a data e o ponto de coleta. Os exemplares obtidos foram submetidos à biometria (medida de comprimento padrão e peso corporal). Em seguida foi realizada a necropsia para coleta dos helmintos. Foi calculado o índice de prevalência, a intensidade média de infecção, teste de comparação " $U$ " de Mann-Whitney entre a prevalência e a intensidade média de infecção, para verificar se existe diferença entre o numero de parasita encontrado em cada campanha foi realizado o teste de Turkey e a relação entre o número de parasitas, peso e comprimento de forma geral e por campanhas.

\section{RESULTADOS E DISCUSSÃO}

Foram analisados 121 exemplares de G. brasiliensis, sendo 26 em julho, 51 em

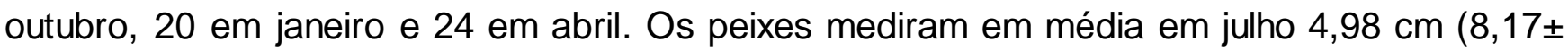
$2,95)$, outubro $6,23 \mathrm{~cm}(11,98 \pm 2,34)$, janeiro $4,61 \mathrm{~cm}(6,97 \pm 2,73)$ e abril $5 \mathrm{~cm}(9,54 \pm 2,23)$ de comprimento e pesaram em média em julho 5,43 g (18,21 \pm 0,92), outubro 8,64 g (51,11 \pm $0,23)$, janeiro $2,51 \mathrm{~g}(8,54 \pm 0,51)$ e abril $6,23 \mathrm{~g}(23,93 \pm 0,35)$. As larvas de Contracaecum foram encontradas na cavidade abdominal tanto livres quanto encasuladas, e também aderidas ao fígado. Houve maior prevalência em janeiro (65\%), o gráfico da variação da prevalência dos parasitas está representado na figura 1 e a variação na intensidade média de infecção, que foi maior em abril $(4,36)$ foi demonstrada na figura 2 . O teste não paramétrico Mann-Whitney " $U$ " mostrou que há diferença significativa entre prevalência e 
Volume 8, Número 2, 2012

Bacias Hidrográficas, Planejamento e

Gestão dos Recursos Hidricos

intensidade média de infecção $(p=0,03)$. Através do teste de Tukey pode observar uma diferença significativa no número de parasitas encontrados em janeiro e abril (Figura 3). A análise geral dos dados mostrou que não existe relação entre o numero de parasitas e o peso $\left(F_{1.56}=0.8547 \mathrm{p}=0.6380\right)$ e o tamanho $\left(F_{1.56}=0.4393 \mathrm{p}=0.5172\right)$ dos indivíduos (Figura 4 A e B). Ao analisar as campanhas separadamente a mesma relação entre numero de parasitas e comprimento foi encontrada para os meses de July $\left(F_{1.14}=0.1469 p=0.7079\right)$; October $\left(\mathrm{F}_{1.16}=1.0581 \mathrm{p}=0.3201\right)$ e April $\left(\mathrm{F}_{1.9}=0.0129 \mathrm{p}=0.9080\right)$ e da mesma maneira para o peso July $\left(F_{1.14}=0.0339 p=0.8505\right)$; October $\left(F_{1.16}=0.5215 p=0.5131\right)$ e April $\left(F_{1.9}=0.0048\right.$ $p=0.9447$ ). Na campanha de January foi a única que observou um relação entre o numero de parasitas e o comprimento $\left(F_{1.11}=5.8828 p=0.0322\right)$ e o peso $\left(F_{1.11}=13.5892 p=0.0038\right)$.

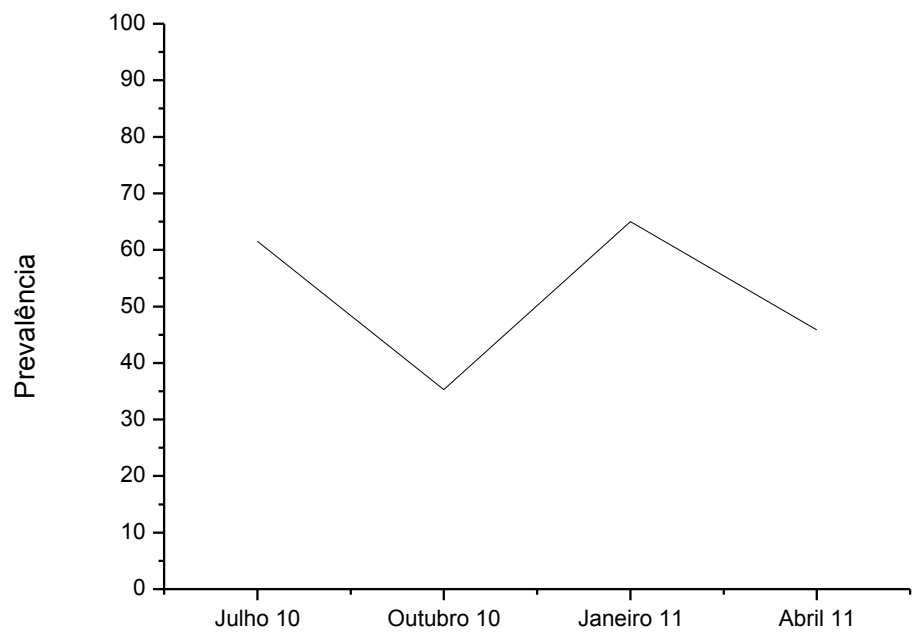

Figura 1 - Variação da prevalência dos Contracaecum em G. brasiliensis na represa do Fazzari nos quatro meses de campanha 


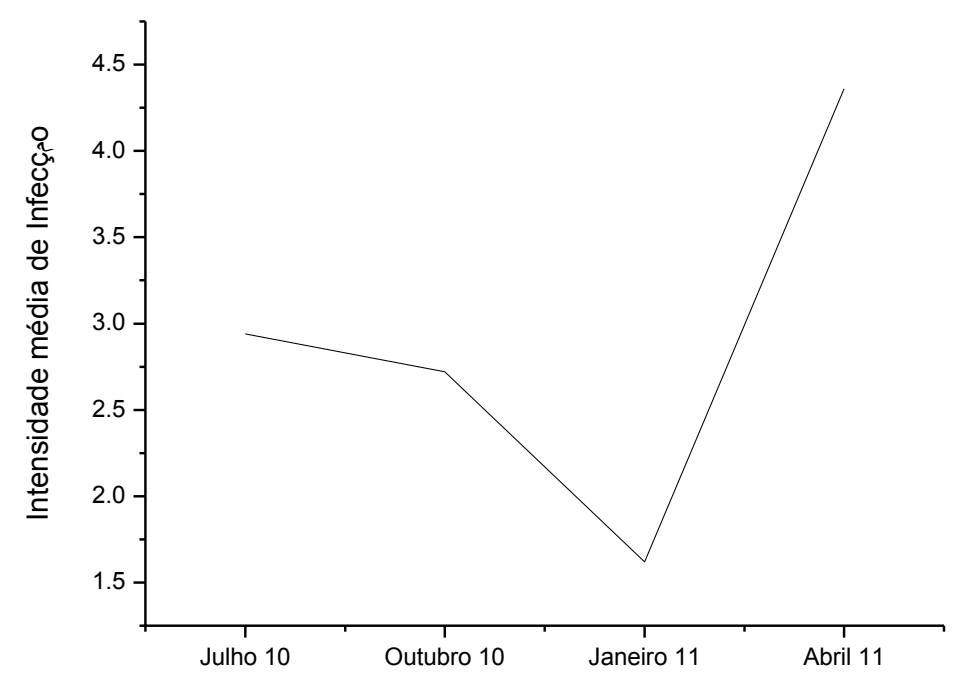

Figura 2 - Variação da Intensidade Média de Infecção dos Contracaecum em G. brasiliensis na represa do Fazzari nos quatro meses de campanha

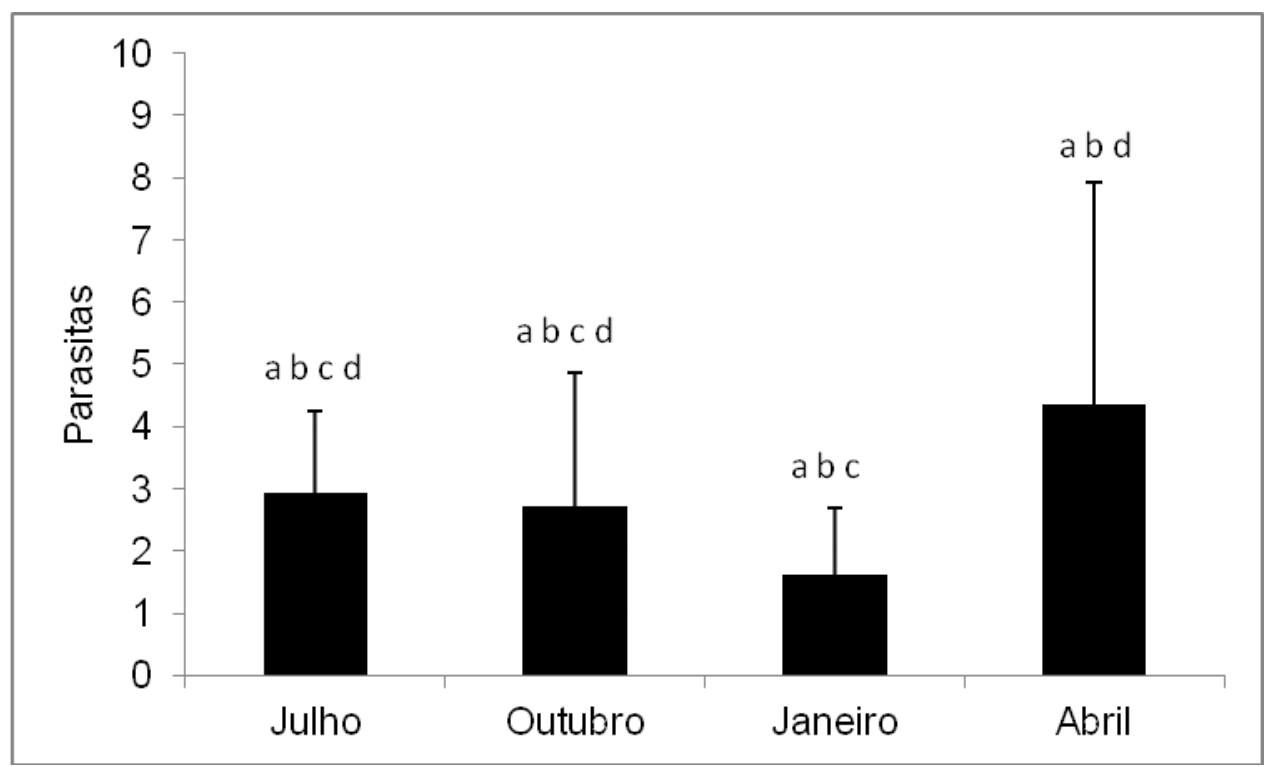

Figura 3 - Relação do número de parasitas entre as campanhas (Julho e Outubro de 2010 e Janeiro e Abril de 2011), as letras diferentes representa diferença significativa $(p<0,05)$ pelo teste de Tukey. 

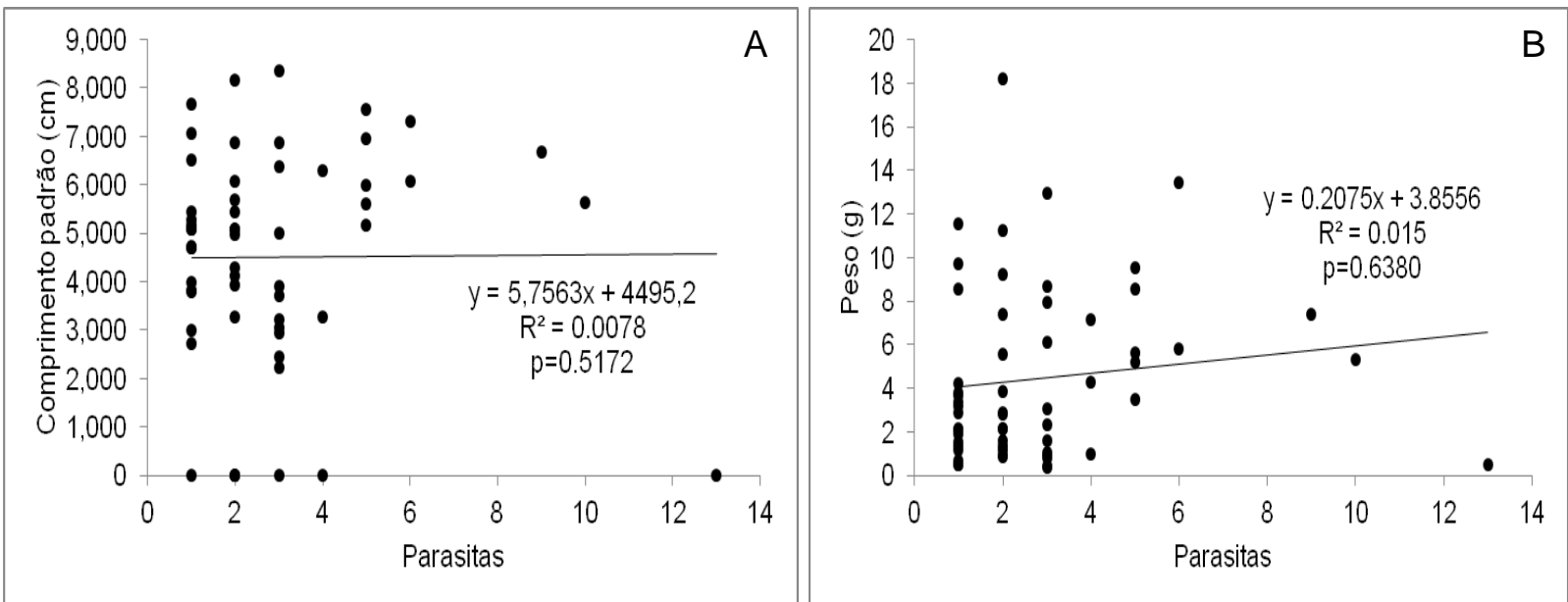

Figura 4 - Relação do número de parasitas encontrados em todas as campanhas (Julho e Outubro de 2010 e Janeiro e Abril de 2011), com o peso (A) e o comprimento (B) dos indivíduos coletados.

Segundo Moreira et al. (2005) hospedeiro e parasita podem se adaptar, em virtude de tal situação, os danos resultantes do parasitismos não são significativos ao hospedeiro, uma vez que o custo do parasitismo é menor que o ganho de energia. Todavia, quando ocorrem grande infestação os parasitas proporcionam danos significativos ao seu hospedeiro (MORAVAC, 1998). Tal fato foi observado em um exemplar de pequenas proporções $(2,49$ $\mathrm{cm}$ e 0,49 g), no qual foram encontradas 13 larvas de Contracaecum encasuladas na cavidade abdominal, a qual ocupava mais de $70 \%$ do local e correspondia a $20 \%$ do peso do indivíduo. De acordo com Zelmer e Arai (1998), Poulin (2000) e Madi e Silva (2005) o aumento da intensidade de infestação é proporcional ao tamanho/idade do hospedeiro e está relacionado com o aumento do volume do item alimentar consumido, uma vez que indivíduos maiores consomem mais alimento, neste estudo não observou uma relação entre o tamanho e intensidade de infestação, este fato pode estar relacionado com o tamanho dos indivíduos coletados, pois só foram capturados indivíduos de pequenas proporções. As larvas de Contracaecum sp não mostraram um padrão estacional de intensidade de infestação, o mesmo foi observado por Martins et al. (2003) e Hamann (2005). 
Volume 8, Número 2, 2012

Bacias Hidrográficas, Planejamento e

Gestão dos Recursos Hídricos

\section{CONCLUSÃO}

- $\quad$ Houve maior prevalência de Contracaecum na campanha de janeiro;

- As altas temperaturas provavelmente aumentam a prevalência de parasitas, uma vez que a maior prevalência de Contracaecum sp em G. brasiliensis ocorreu no período quente do verão no mês de Abril.

- A menor infestação também foi ocorreu o período de verão (Janeiro), e infestação máximo ocorreu no outono e no período seco;

- $\quad$ Houve correlação positiva entre o número de parasitas e o comprimento e o peso no verão;

- $\quad$ Existe diferença significativa entre prevalência e intensidade média de infecção.

\section{AGRADECIMENTOS}

Os autores agradecem ao Programa de Pós-graduação em Ecologia e Recursos Naturais da Universidade Federal de São Carlos SP e ao CAPES pelo financiamento deste trabalho. Agradecemos ao professor Dr. Reinaldo e as mestrandas Lidiane e Aline do Departamento de Parisitologia, da Universidade Estadual de São Paulo Botucatu pela identificação dos nematodas.

\section{REFERÊNCIAS}

ALBUQUERQUE, L. B. Entomofauna aquatica do litoral de dois reservatórios da região de São Carlos - SP. São Carlos - SP, UFSCar, 94p. Dissertação (Mestrado), 1990.

GUIDELLI, G.M.; ISSAC, A.; TAKEMOTO, R.M.; PAVANELLI, G.C. Endoparasite infracommunities of Hemisorubim platyrhynchos (Valenciennes, 1840) (Pisces: Pimelodidae) 
of the Baía river, Upper Paraná River floodplain, Brazil: specific composition and ecological aspects. Brazilian Journal of Biology, v.63, n. 2, p. 261-268, 2003.

HAMANN, M.I. Aspectos ecológicos de la relación parasitaria entre larvas de Contracaecum sp. (Nematoda, Anisakidae) y Serrasalmus spilopleura Kner, 1860 (Pisces, Characidae) en poblaciones naturales del nordeste argentino. Boletín chileno de parasitología, Santiago, v. 54, n. 3-4, jul. 1999. Disponivel em $<$ http://www.scielo.cl/scielo.php?script=sci_arttext\&pid=S036594021999000300007\&lng=es\&nrm=iso $>$. acessado em 29/09/011. doi: 10.4067/S036594021999000300007.

MADI, R. R. Utilização dos helmintos parasitas de Geophagus brasiliensis (Quoy \& Gairmard, 1824) (Cichlidae; Perciformes) como indicadores ambientais. PhD Tese, Campinas, 2005.

MADI, R.R. SILVA, M.S.R. Contracaecum Railliet \& Henry, 1912 (Nematoda, Anisakidae): o parasitismo relacionado à biologia de três espécies de peixes piscívoros no reservatório do Jaguari, SP. Revista Brasileira de Zoociências, v.7 n. 1, p.15-24, 2005.

MARTINS, M.L.; ONAKA, E.M.; FENERICK, J.J. Larval Contracaecum sp. (Nematoda: Anisakidae) in Hoplias malabaricus and Hoplerythrinus unitaeniatus (Osteichthyes: Erythrinidae) of economic importance in occidental marshlands of Maranhao. Brazilian Veterinary Parasitology, v. 127, p. 51-59, 2005.

MARTINS, M.L.; SANTOS, R.S.; TAKAHASHI, H.K.; MARENGONI, N.G.; FUJIMOTO, R.Y. Infection and susceptibility of three fish species from the Paraná River, Presidente Epitácio, State of São Paulo, Brazil, to Contracaecum sp. larvae (Nematoda: Anisakidae) Acta Scientiarum. Animal Sciences, Maringá, v. 25, no. 1, p. 73-78, 2003.

MORAVEC, F. Nematodes of freshwater fishes of the Neotropical region. Praga, Academia, 1998.

MOREIRA, S.T.; ITO, K.F.; TAKEMOTO, R.M.; PAVANELLI, G.C. Ecological aspects of the parasites of Iheringichthys labrosus (Lütken, 1874) (Siluriformes: Pimelodidae) in reservoirs of Paraná basin and upper Paraná floodplain, Brazil. Acta Scientiarum Biological Sciences, v. 27, n. 4, p. 317-322, 2005.

PAVANELLI, G.C.; MACHADO, M.H.; TAKEMOTO, R.M.; GUIDELLI, G.M.; LIZAMA, M.A.P. In: THOMAZ, S.M.; AGOSTINHO, A.A.; HAHN, N.S. (Eds.). The upper Paraná river and its floodplain: physical aspects, ecology and conservation. Netherlands: Backhuys Publishers, Leiden, p. 309-329, 2004. 
POULIN, R. Variation in intraspecific relationship between fish length and intensity of parasitic infection: biological and statistical causes. Journal Fish Biology, 56: 123-127, 2000.

TORRES, P.; VALDIVIESO, J.; SCHALTTER, R.; MONTEFUSCO, A.; REVENGA, J.; MARÍN, F.; LAMILLA, J.; RAMALTO, G. Infection by Contracaecum rudolphii (Nematoda: Anisakidae) in the Neotropical cormorant Phalacrocorax brasilianus, and fishes from the estuary of the Valdivia river, Chile. Studies on Neotropical Fauna and Environment, v. 35, n. 2, p. 101-108, 2000.

VICENTE, J.J.; RODRIGUES, H.O.; GOMES, D.C. Nematóides do Brasil - 1ª parte: Nematóides de peixes. Atlas da Sociedade de Biologia do Rio de Janeiro, v. 25, p. 1-79, 1995.

ZELMER, D.A.; ARAI, H.P. The contributions of host age and size to the aggregated distribution of parasites in yellow perch, Perca flavecens, from Garner Lake, Alberta, Canada. The Journal of Parasitology, v. 84, n. 1, p. 24-28, 1998. 